\title{
$\Lambda$ CDM halo density profiles: where do actual halos converge to NFW ones?
}

\author{
G. Gentile ${ }^{1,2}$, C. Tonini ${ }^{1}$, and P. Salucci ${ }^{1}$ \\ 1 SISSA, via Beirut 4, 34014 Trieste, Italy \\ e-mail: ggentile@unm.edu \\ ${ }^{2}$ University of New Mexico, Department of Physics and Astronomy, 800 Yale Blvd NE, Albuquerque, NM 87131, USA
}

Received 3 May 2006 / Accepted 12 January 2007

\section{ABSTRACT}

\begin{abstract}
Aims. We present an analysis of 37 high-quality extended rotation curves that highlight the existence of a new discrepancy (or a new aspect of an old discrepancy) between the density profiles predicted by the $\Lambda$ cold dark matter $(\Lambda \mathrm{CDM})$ theory and the actual distribution of dark matter in galaxies.

Methods. We compare the predicted face-value density vs. enclosed mass relationship, at large distances, to the observational data at the last measured radii of the rotation curves and in two whole rotation curves of high quality. A further analysis is performed by studying a relation, inbuilt in $\Lambda \mathrm{CDM}$, that links, at radius $R$, the enclosed halo mass $M_{\mathrm{NFW}}(R)$ and its density $\rho(R)$ in a way that is independent of the mass of the virialised object.

Results. We find that the predicted density vs. enclosed mass relationship has a systematic offset with respect to the observational data. In test case extended rotation curves, at their last measured point, the predicted NFW densities are up to a factor 3 lower than those derived from the kinematics. Moreover, the abovementioned relation, inbuilt in $\Lambda$ CDM, does not hold for the objects of our sample. Such a new outer discrepancy is different and maybe complementary with respect to the core/cusp issue, for which the NFW densities turn out to be higher than those observed, and it seems to imply a global mass rearrangement of a pristine NFW- $\Lambda$ CDM halo.
\end{abstract}

Key words. galaxies: kinematics and dynamics - cosmology: dark matter - galaxies: structure

\section{Introduction}

The study of disc galaxies' rotation curves has been one of the most successful tools for investigating the dark matter phenomenon in galaxies. Recently, the attention has been focused on the distribution of dark matter in galaxies, as inferred from the rotation curves, in particular the comparison between the predictions of standard $\Lambda$ cold dark matter $(\Lambda \mathrm{CDM})$ theory of structure formation and observations (e.g., Salucci \& Burkert 2000; Borriello \& Salucci 2001; de Blok \& Bosma 2002; Weldrake et al. 2003; Swaters et al. 2003; Simon et al. 2005; Gentile et al. 2004, 2005, 2007). More specifically, the $\Lambda$ CDM theory predicts that dark matter halos have a specific density distribution that follows the well-known NFW (Navarro et al. 1996) profile:

$\rho_{\mathrm{NFW}}(R)=\frac{\rho_{\mathrm{s}}}{\left(R / r_{\mathrm{s}}\right)\left(1+R / r_{\mathrm{s}}\right)^{2}}$,

where $r_{\mathrm{s}}$ and $\rho_{\mathrm{s}}$ are the characteristic radius and density of the distribution. The latter is given by:

$\rho_{\mathrm{s}}=\frac{\Delta}{3} \frac{c^{3}}{\ln (1+c)-\frac{c}{1+c}} \rho_{\mathrm{c}}$,

where $\rho_{\mathrm{c}}$ is the critical density of the universe and $\Delta$ is the virial overdensity (see Bryan \& Norman 1998). $r_{\mathrm{s}}$ and $\rho_{\mathrm{s}}$ are related to each other (e.g., Wechsler et al. 2002), so Eq. (1) is rather a one-parameter family of profiles, where the following relations link the virial mass $M_{\text {vir }}$ to the concentration parameter $c\left(=r_{\mathrm{vir}} / r_{\mathrm{s}}\right.$, where $r_{\mathrm{vir}}$ is the virial radius), $r_{\mathrm{s}}$, and $\rho_{\mathrm{s}}$, at redshift $z=0$, and adapting the relations (similarly to Gnedin et al. 2006; and Dutton et al. 2006) to the cosmological parameters from the WMAP third year results (Spergel et al. 2006):

$c \simeq 13.6\left(\frac{M_{\mathrm{vir}}}{10^{11} M_{\odot}}\right)^{-0.13}, r_{\mathrm{s}} \simeq 8.8\left(\frac{M_{\mathrm{vir}}}{10^{11} M_{\odot}}\right)^{0.46} \mathrm{kpc}$.

The virial radius $r_{\mathrm{vir}}$ can then be derived from $r_{\mathrm{vir}}=c r_{\mathrm{s}}$. In $\Lambda \mathrm{CDM}$, at least in a statistical sense, once the radius and the mass at that radius are fixed, the parameter describing the mass distribution (usually the virial mass $M_{\text {vir }}$ ) is also known. On the other hand, a quite remarkable number of observations show that NFW profiles, displaying an inner "cusp", are inconsistent with data. In fact, the latter indicate profiles with a different characteristic, a central density "core", i.e., a region where the dark matter density remains approximately constant. A number of studies cast doubts on the reliability of the mass modelling procedure and the data analysis (van den Bosch et al. 2000; Swaters et al. 2003; Hayashi \& Navarro 2006; Valenzuela et al. 2007) of some galaxies. The debate is still on, despite the fact that most concerns are now overcome (Gentile et al. 2004, 2005, 2006; de Blok et al. 2003; de Blok 2005).

The simplest example of a cored halo profile is the pseudoisothermal (PI, van Albada et al. 1985):

$\rho_{\mathrm{PI}}(R)=\frac{\rho_{0}}{1+R^{2} / R_{\mathrm{C}}^{2}}$,

where $R_{\mathrm{C}}$ is the core radius and $\rho_{0}$ is the central density, that results about one order of magnitude lower than $\Lambda$ CDM predictions (Donato et al. 2004; Gentile et al. 2004 2005, and 
references therein). However, the above works did focus on the comparison between $\Lambda \mathrm{CDM}$ and observations in the inner parts of the galaxies, while it is also of extreme importance to investigate the outer density distribution, where $\Lambda \mathrm{CDM}$ halos have an equally strong feature: the transition between a $\rho \propto R^{-1}$ to a $\rho \propto R^{-3}$ regime. In this paper, outer and inner regions have a baryonic perspective. The former refers to a region inside 1-2 stellar disc scale lengths $R_{\mathrm{D}}$, the latter to that extended out to $\gtrsim 3-5 R_{\mathrm{D}}$ and characterised by the HIdisc. Both regions, however, must be considered inner regions with respect to the DM distributions. In other words, is the discrepancy between theoretical predictions and data present only in the inner parts of a galaxy, and therefore the profiles converge to the NFW one at larger radii, or is there a more global discrepancy extending beyond the NFW halo $R^{-1}$ regime, as was also suggested by McGaugh et al. (2007)?

Note that the rotation curves at large radii, i.e., for $R \gg$ $R_{\mathrm{D}}$, provide a very good measure of densities and of enclosed dark masses. In fact, at these distances, the contribution to the gravitational potential due to gas and stars is very small and the uncertainties on the accurateness/uniqueness of the dark-toluminous mass decomposition play a very minor role: it is the rotation curve itself that essentially measures the physical quantities $M_{\mathrm{h}}(R) \simeq G^{-1} V^{2}(R) R$ and $\rho_{\mathrm{h}}(R) \simeq 1 /\left(4 \pi R^{2}\right) \mathrm{d} M_{\mathrm{h}}(R) / \mathrm{d} R$. Conversely, in the inner regions $\left(R<R_{\mathrm{D}}\right)$, usually, a complex dark-luminous mass modelling is needed to decompose the circular velocity into the (possibly equally important) dark and luminous contributions (see, e.g., Barnes et al. 2004).

\section{Samples and methods of investigation}

In the present paper we investigate the outer dark matter distribution mostly resorting to two samples, heterogeneous in mass, covering about 3 orders of magnitude: 1) the sample of highquality rotation curves selected by Donato et al. (2004), discarding the 4 galaxies with the smallest extension relative to the disc exponential scale length $R_{\mathrm{D}}$ and adding the galaxies: DDO 47 (Salucci et al. 2003; Gentile et al. 2005) and ESO 287-G13 (Gentile et al. 2004); 2) a sample with rotation curves selected from the literature fulfilling the requirements of a) reaching out to at least $30 \mathrm{kpc}$, or out to 6 disc scale lengths, or b) with the final velocity being above $250 \mathrm{~km} \mathrm{~s}^{-1}$. In this way, the curves were sufficiently extended to map a region of the halo density profile not affected by the central slope, nor massively affected by the presence of the disc. It results that we investigate regions of halos corresponding to galactocentric distances out to $5 \%$ up to about $35 \%$ (the case of NGC 9133) of the virial radius: these zones are beyond the influence of the cusp, and still well into the central part of the dark matter halo. We selected only rotation curves that were regular out to the last measured radius $R_{\mathrm{f}}$ (whose average value is about $24 \mathrm{kpc}$ ), obtaining a sample of 37 galaxies. In Table 1 the selected galaxies of sample 2 are listed, together with the corresponding references. For a number of galaxies the mass decomposition between the luminous and dark components of the velocity was obtained from the literature; we marked them with an asterisk in Table 1. For the others, we used the method of Persic \& Salucci (1990), see Appendix A.

The rotation curves of sample 1 have been selected and successfully used (Donato et al. 2004) to investigate the core radius issue, i.e., an issue that needs more accurate data than the issue we want to tackle here. Since the resulting halo rotation curves of the galaxies of the sample are very well fitted by the PI profile, we will take it as representative of the dark matter haloes around galaxies. From our sample 1 we select
Table 1. Selected galaxies with their references. The asterisk indicates that the original work also provided the dark-luminous decomposition of the rotation curve.

\begin{tabular}{ll}
\hline \hline Galaxy & Reference \\
\hline NGC 289* & Walsh et al. (1997) \\
NGC 1068 & Sofue et al. (1999) \\
NGC 1097 & Sofue et al. (1999) \\
NGC 1232* & van Zee \& Bryant (1999) \\
NGC 3198* & Blais-Ouellette et al. (2001) \\
NGC 3726 & Verheijen \& Sancisi (2001) \\
NGC 4123* & Weiner et al. (2001) \\
NGC 5055 & Sofue et al. (1999) \\
NGC 5236 & Sofue et al. (1999) \\
UGC 5253 & Noordermeer et al. (2004) \\
NGC 5985 & Blais-Ouellette et al. (2004) \\
NGC 6946* & Carignan et al. (1990) \\
NGC 7331* & Bottema (1999) \\
UGC 9133* & Noordermeer et al. (2004) \\
\hline
\end{tabular}

DDO 47 and ESO 287-G13, as the best examples to individually show the outer NFW $\Lambda$ CDM-data discrepancy. Then, we use the combined samples 1-2 to compare, at the outermost radii, the NFW $\Lambda$ CDM predictions, obtained by a newly discovered structural $\Lambda \mathrm{CDM}$ relation, with the values of the mass, radius, and density of the DM halos around galaxies.

\section{The new dark matter density discrepancy from the extended rotation curves of ESO 287-G13 and DDO 47}

We analyse in detail the density profiles of the DM halos for the two best cases in our sample: DDO 47 (Salucci et al. 2003; Gentile et al. 2005) and ESO 287-G13 (Gentile et al. 2004). Note that in the present study, differently from the original papers, the aim is to show the existence of an outer observations/theory discrepancy, rather than to investigate the inner cusp/core issue: therefore we will analyse the data in a different but proper way. For consistency with the detailed analysis made in the original papers, in this section we used the relation between $c$ and $M_{\mathrm{vir}}$ given by Bullock et al. (2001) and Wechsler et al. (2002) instead of Eq. (3).

\subsection{Actual dark matter halos}

We follow Salucci et al. (2003) and Gentile et al. (2004), the density distribution $\rho_{\mathrm{h}}(R)$ of the dark matter halo in these galaxies is given by: $\rho_{\mathrm{h}}(R)=\rho_{\mathrm{PI}}(R)$ (see Eq. (4)). Notice that in both objects we have also considered a Burkert halo (Burkert 1995), and found results coincident with the PI halo.

\subsection{NFW halos}

Let us assume that:

$M_{\mathrm{NFW}}\left(R_{\mathrm{f}}\right)=M_{\mathrm{PI}}\left(R_{\mathrm{f}}\right)$,

where $M_{\mathrm{PI}}\left(R_{\mathrm{f}}\right)$ is the mass inside $R_{\mathrm{f}}$ that we derive from the above mass model. Equation (5) allows us to derive the values for $c$ and $M_{\mathrm{vir}}: 18.4$ and $6 \times 10^{10} M_{\odot}$ for DDO 47, and 13.3 and $7 \times 10^{11} M_{\odot}$ for ESO 287-G13. Let us anticipate that this assumption is very conservative; in fact, if the mass equality expressed by Eq. (5) occurs at radii smaller than $R_{\mathrm{f}}$, the result we claim here will be even more prominent; on the other hand, if 

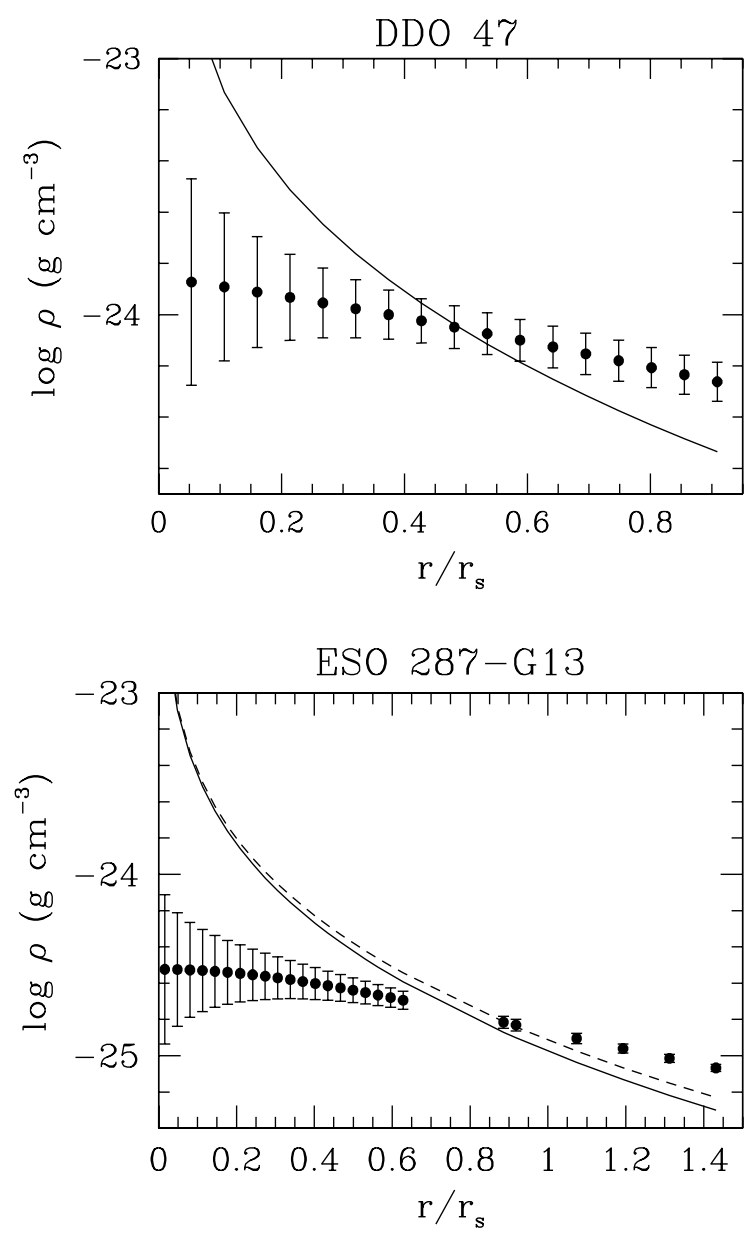

Fig. 1. DM density distributions; filled circles represent the DM density inferred by the rotation curves. Solid lines correspond to the NFW solution with $M_{\mathrm{NFW}}\left(R_{f}\right)=M_{\mathrm{PI}}\left(R_{\mathrm{f}}\right)$, which give, for DDO 47, $\left(r_{\mathrm{s}}\right.$, $\left.M / L_{\mathrm{B}} ; R_{\mathrm{D}}\right)=(5.5 \mathrm{kpc}, 0.5 ; 0.5 \mathrm{kpc})$, and for ESO $287-\mathrm{G} 13,\left(r_{\mathrm{s}}, M / L_{\mathrm{I}}\right.$; $\left.R_{\mathrm{D}}\right)=(17.4 \mathrm{kpc}, 1.8 ; 3.3 \mathrm{kpc})$. The dashed line corresponds to the "bestfit" NFW solution, $\left(r_{\mathrm{s}}, M / L_{\mathrm{I}}\right)=(19.4 \mathrm{kpc}, 0.7)$. See text for the (conservative) estimate of the uncertainties.

the mass equality occurs at radii larger than $R_{\mathrm{f}}$, a discrepancy from $R=0$ out to $R=R_{\mathrm{f}}$ will be set by definition. This assumption also has the desirable by-product, in the NFW framework, of implying reasonable values for the stellar mass-to-light $(M / L)$ ratios. Given the importance of a careful analysis, we have also considered a different implementation of the NFW halo + disc + gas mass modelling: in ESO 287-G13 we have fitted the rotation curve with this mass model by leaving $M / L$ and $M_{\text {vir }}$ as free parameters; this latter procedure, shown in Fig. 1, yields very similar results with respect to those obtained by means of Eq. (5).

\subsection{Test for ESO 287-G13 and DDO 47}

In Fig. 1 we compare the actual dark matter density $\rho_{\mathrm{PI}}(R)$ and $\rho_{\mathrm{NFW}}(R)$ at the radii where the rotation curves were measured. A conservative estimate of the uncertainty on the density was derived from the mass modelling uncertainties. In fact $20 \%$ uncertainties in $\rho_{0}$ and $R_{\mathrm{C}}$ give an average uncertainty on the density of about $25 \%$. A rigorous derivation goes beyond the scope of this paper. Then, it is clear that the density is not determined with the same accuracy at each radius. In fact dark matter is not the dominant kinematic term in the inner parts, and therefore the density uncertainty is larger.

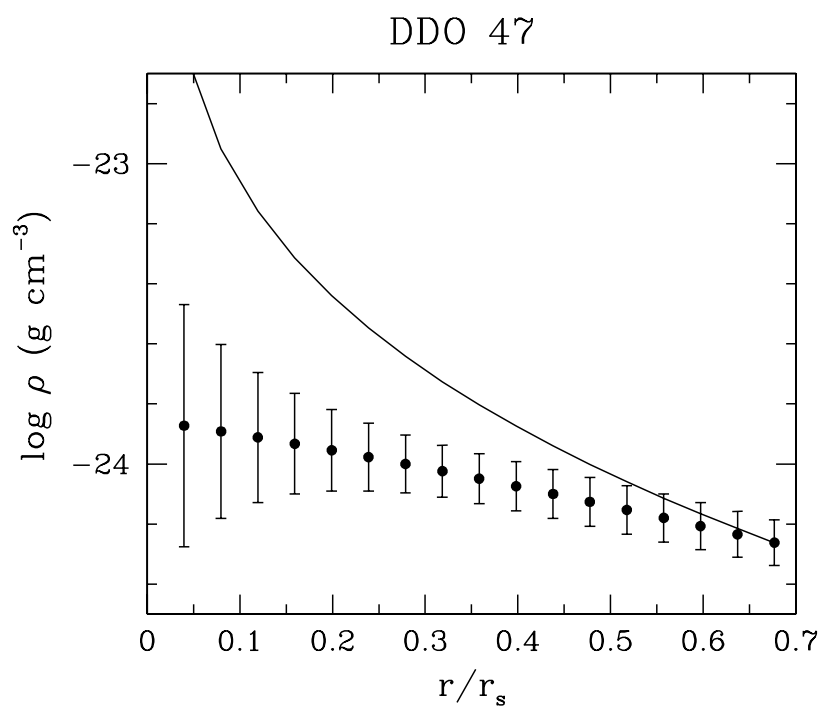

Fig. 2. Density profiles of DDO 47 assuming $\rho_{\mathrm{NFW}}\left(R_{\mathrm{f}}\right)=\rho\left(R_{\mathrm{f}}\right)$. See Fig. 1 for the explanation of the symbols.

We see the well-known (though debated in some case) cuspcore discrepancy at $R \rightarrow 0$, but at larger radii we now realise that the difference in densities changes sign and that the "inner" discrepancy is now reversed: in both analyses, the NFW halo densities, from a certain radius onward, are lower than the dark matter halo density. Note that such a discrepancy was also present in some of the analyses made in previous investigations (e.g., Blais-Ouellette et al. 2001; Borriello \& Salucci 2001; de Blok \& Bosma 2002), but it was not claimed explicitly, nor was it investigated whether some combination of $c$ and $M_{\text {vir }}$ had it made possible for $\rho_{\mathrm{NFW}}$ to converge to the actual density inside the region mapped by the rotation curve data. In the present paper we instead claim that the situation can be described by one of these two possibilities: 1) $\rho_{\mathrm{NFW}}(R)$ at any radius is systematically higher than the estimated density (so it will disagree in the core region, but will be compatible with the outermost density, see Fig. 2) and the data vs. prediction discrepancy will extend to several disc scale lengths; 2) the discrepancy is "bivariate": actual halos (in comparison to predicted halos) have a density deficit in the inner regions, but an excess in the outer ones. We consider the second possibility as more likely since it implies stellar $M / L$ ratios compatible with the predictions of stellar population synthesis models (Bell et al. 2003; Gentile et al. 2004). For instance, in the case of DDO 47, imposing $\rho_{\mathrm{NFW}}(R)=\rho(R)$ leads to a best-fit $M / L$ ratio of 0 ; on the other hand, with the second possibility one gets a $B$-band $M / L=0.7$, within the range $0.5-0.8$ arising from the observed $B-V$ colour and the predictions of stellar population synthesis models (Bell et al. 2003). Let us stress that the availability of data at large distances is a crucial point: in these galaxies we just reach the radius where the NFW density decreases in an appreciable way. Compared to the discrepancy in the inner regions of galaxies, extremely evident and as wide as an order of magnitude, the outer discrepancy is less spectacular but not less important.

\section{The new density discrepancy from a new NFW halo phenomenology}

A main property of the distribution of NFW-CDM haloes is that $M_{\mathrm{NFW}}(R)=f\left(R, M_{\mathrm{vir}}, c\left(M_{\mathrm{vir}}\right)\right)$, while $\rho_{\mathrm{NFW}}(R)=$ $h\left(R, M_{\mathrm{vir}}, c\left(M_{\mathrm{vir}}\right)\right)$. From this it follows that: $M_{\mathrm{NFW}}(R)=$ $\mathcal{G}\left(R, \rho_{\mathrm{NFW}}(R), M_{\mathrm{vir}}\right)$, that is, at a fixed radius, the density and 


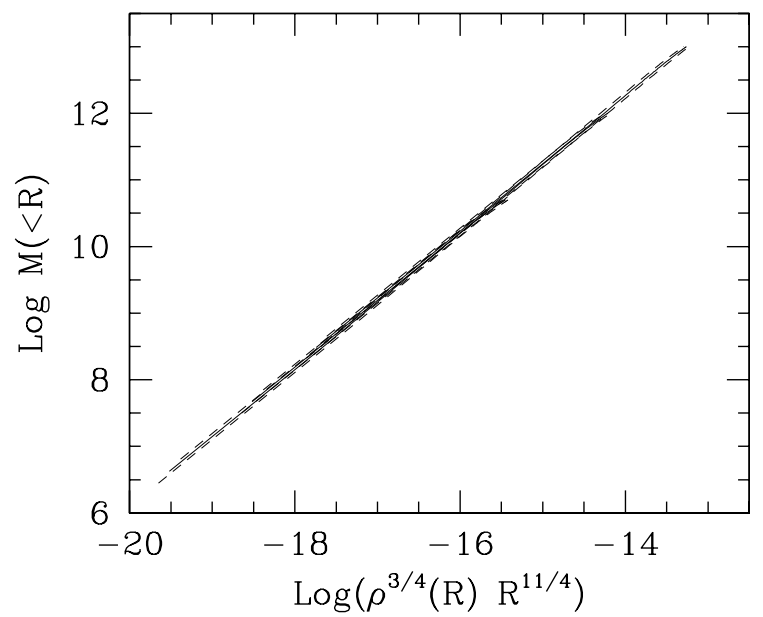

Fig. 3. The $M-R-\rho$ relation: the (overlapping) solid lines refer to the $\Lambda \mathrm{CDM}$ haloes, for 3 different virial masses $\left(5 \times 10^{10} M_{\odot}, 1 \times 10^{12} M_{\odot}\right.$ and $1 \times 10^{13} M_{\odot}$ ) and the 3 corresponding concentration parameters $c$ according to Wechsler et al. (2002) (18.7, 12.7 and 9.4, respectively). The dashed lines correspond to the $\pm 1-\sigma$ uncertainty in $c$ taken from Wechsler et al. (2002). Radii range from $0.001 r_{\mathrm{vir}}$ to $r_{\mathrm{vir}} . M(<R)$ is in $M_{\odot}, \rho(R)$ in $\mathrm{g} \mathrm{cm}^{-3}$ and $R$ in $\mathrm{kpc}$.

mass are related, though in principle in a different way in galaxies of different $M_{\text {vir }}$. The above provides us with a convenient way to present the structural properties of NFW halos, according to which observational data can be compared with the theory without knowing the virial masses of the objects. This is crucial because of the poor fitting performance of the NFW fits to rotation curves, which often prevents even a rough estimate of this quantity. The relation we find (hereafter called the $M-R-\rho$ relation) is valid for any $M_{\text {vir }}$; this can be easily seen in Fig. 3 by plotting it for 3 different virial (total) masses $\left(5 \times 10^{10} M_{\odot}\right.$, $1 \times 10^{12} M_{\odot}$ and $1 \times 10^{13} M_{\odot}$ ) and concentration parameters (see Eq. (2)), for radii ranging from $0.001 r_{\text {vir }}$ to $r_{\text {vir }}$ the relation takes the form (see Fig. 3):

$\log M_{\mathrm{NFW}}=\frac{3}{4} \log \rho_{\mathrm{NFW}}(R)+\frac{11}{4} \log R+26.17$,

where $M_{\mathrm{NFW}}$ is in $M_{\odot}, R$ in $\mathrm{kpc}$ and $\rho_{\mathrm{NFW}}$ in $\mathrm{g} \mathrm{cm}^{-3}$. The $M-$ $R-\rho$ relation holds for more than 6 orders of magnitude in mass, and it is valid, for real cases, for any galactic halo at any radius. The scatter in $c, 3$ orders of magnitude in radii, and a factor 200 in $M_{\text {vir }}$ introduce a negligible $(\lesssim 0.05$ dex) scatter through the 6 orders of magnitude in $M_{\mathrm{NFW}}(R)$.

The relation follows from the structural properties of NFW halos. It can be derived mathematically, but its physical meaning is reported at the origin of Eqs. (1) and (3). Let us write the ratio between the enclosed mass $M_{\mathrm{NFW}}(<R)$ of an NFW halo at radius $r$ (where the density is $\rho_{\mathrm{NFW}}(R)$ ) and a uniform sphere of radius $r$ and density $\rho_{\mathrm{NFW}}(R)$ :

$\frac{M_{\mathrm{NFW}}(<r)}{4 / 3 \pi r^{3} \rho_{\mathrm{NFW}}(R)}=\frac{3(1+c x)^{2}}{c^{2} x^{2}} \cdot\left(\ln (1+c x)-\frac{c x}{1+c x}\right)$.

On the other hand, from Eq. (6) we have: $\frac{M_{\mathrm{NFW}}(<r)}{4 / 3 \pi r^{3} \rho_{\mathrm{NFW}}(R)} \propto$ $\left(\rho_{\mathrm{NFW}}(R) R\right)^{-1 / 4}$. Then, recalling that $\left(\rho_{\mathrm{NFW}}(R) R\right)^{-1 / 4}=\frac{(1+c x)^{1 / 2}}{\left(\rho_{\mathrm{s}} r_{\mathrm{s}}\right)^{1 / 4}}$, one finds that the $M-R-\rho$ relation is a way of expressing the approximation (valid for $0<x<1$ ):

$(1+c x)^{1 / 2} \propto A \frac{3(1+c x)^{2}}{\left(c^{2} x^{2}\right)}\left(\ln (1+c x)-\frac{c x}{1+c x}\right)$.

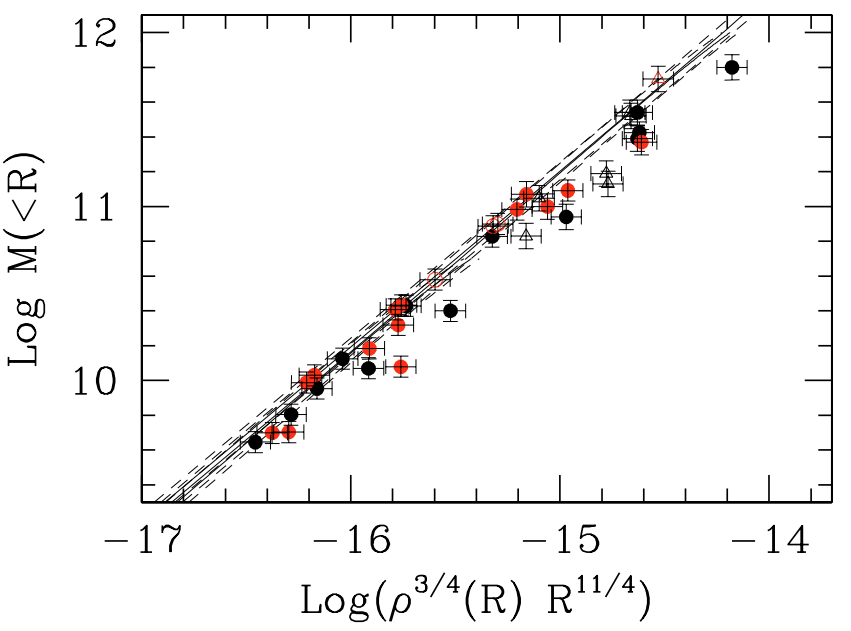

Fig. 4. The same as Fig. 3, zoomed to the radial range from $1 \%$ to $20 \%$ of the virial radius. Filled circles are the observational data of samples 1 and 2 at $R_{\mathrm{f}}$. The three edge-on galaxies are denoted by empty circles. Empty triangles denote the objects of sample 2 without published mass modelling. Red symbols are isolated halos and black symbols are subhalos, according to our definition in the text.

We compared the predicted $M-R-\rho$ relation with the measurements of 37 galaxies. The result (Fig. 4) is that the observational points at $R_{\mathrm{f}}$ are systematically offset compared to the $M-R-\rho$ relation. The mean offset of $\sim 0.1 \mathrm{dex}$ is solid (but note that larger offsets are also observed); in fact, the error propagation analysis shows that a $3 \%$ uncertainty on the measure of the circular velocity and a 0.05 uncertainty on the logarithmic gradient $\frac{\operatorname{dlog} V(R)}{\operatorname{dlog} R}$ yield uncertainties of the order of 0.025 dex in $M$ and 0.06 dex in $\rho$. The uncertainties on the objects' distances are not included since they only induce random errors. At $R_{\mathrm{f}}$, for a given enclosed mass $M\left(<R_{\mathrm{f}}\right)$, the observed density $\rho_{\mathrm{PI}}\left(R_{\mathrm{f}}\right)$ is higher than that of NFW haloes that match $M\left(<R_{\mathrm{f}}\right)$. The density discrepancy runs up to a factor $\sim 3$ and it is significant in that the adopted procedure of matching the CDM mass to the observed one is a conservative one. The predicted halo profiles are based on a larger number of halos than the present sample of 37 galaxies, so selection or systematic effects cannot be completely excluded, even if the 37 galaxies span a large range of maximum velocities, Hubble types, and environments.

We tested whether the environment has an effect on our results: we distinguished between galaxies in "isolated" halos and galaxies in "subhalos". A straightforward definition does not exist, so we defined those galaxies that either belong to a known group of galaxies or that have a larger companion within $30 R_{25}$ and $400 \mathrm{~km} \mathrm{~s}^{-1}$ as "subhalos". The other galaxies were labelled as "isolated". A more detailed investigation of the effect of environment goes beyond the scope of our paper, especially because of the heterogeneity of the data we consider here. Qualitatively, there are no obvious differences between the different subsamples, even though the predicted difference between the concentrations of halos and subhalos (Bullock et al. 2001) cannot be excluded.

Edge-on galaxies may represent a potential problem, as the $\mathrm{H} \alpha$ rotation curves could be affected by extinction (Bosma et al. 1992) and the HI rotation curves might suffer from unaccountedfor projection effects and from the possible lack of gas on the line of nodes. In Fig. 4 we have plotted the three edge-on galaxies (i.e., with an inclination larger than $85^{\circ}$ ) of our sample with a triangle; we realise that these galaxies do not lie in any peculiar region of the plane and they are not more discrepant than the 


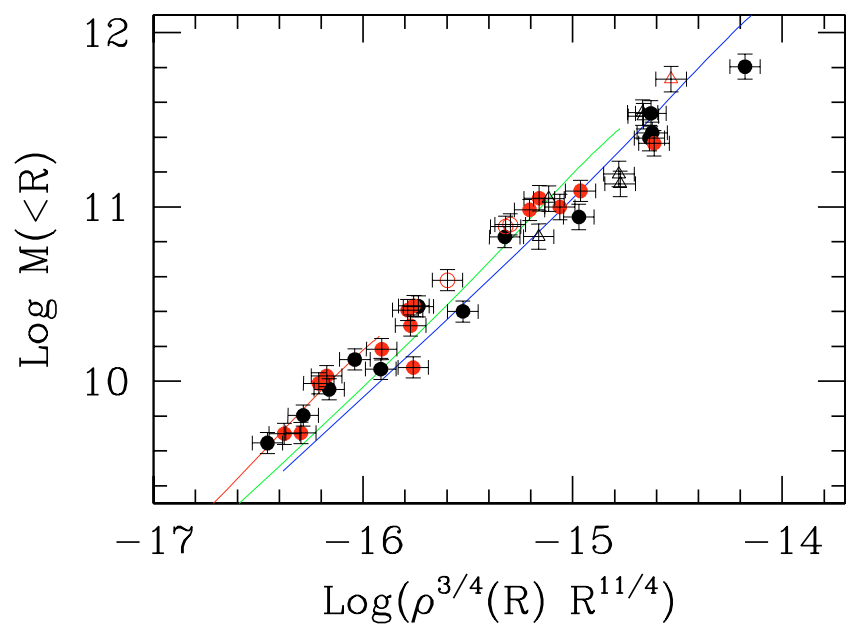

Fig. 5. The same as Fig. 4, but showing that Burkert halos (Salucci \& Burkert 2000) predict a mass-dependent $\operatorname{Mvs}\left(\rho^{a} R^{b}\right)$ relation in good agreement with observations. Symbols are the same as Fig. 4, and the 3 lines correspond to 3 different virial masses $\left(5 \times 10^{10} M_{\odot}, 1 \times 10^{12} M_{\odot}\right.$, and $1 \times 10^{13} M_{\odot}$, see text).

other galaxies. The same holds for the galaxies from sample 2 for which the mass model was obtained as described in the previous section.

Baryons are expected to affect the density distribution of dark matter, but the effects are far from being clear: the best studied process is adiabatic contraction (Blumenthal et al. 1986; Gnedin et al. 2004; Sellwood \& McGaugh 2005; Gustafsson et al. 2006), which would make the halos more centrally concentrated, but mechanisms with opposite effects have also been studied, such as adiabatic expansion (Dutton et al. 2006) or dynamical friction (Tonini et al. 2006). Hence, in the $\Lambda$ CDM halos considered here we did not take the effect of baryons into account, since their effect on the distribution of dark matter is still poorly understood. Also, when gas cools, baryons are expected to make dark matter halos more spherical (Kazantzidis et al. 2004). In Fig. 6 we plotted the baryonic mass of the galaxies of our sample with mass decompositions vs. their distance from the relation in Eq. (6): no clear correlation is observed, meaning that the discrepancy we report in the present paper is not straightforwardly related to the amount of baryons present in the galaxy, or that effects such as uncertainties in the mass distribution create a large scatter in Fig. 6 . We also note that in the galaxies studied here, $R_{\mathrm{f}} \gg R_{\mathrm{D}}$, i.e., $R_{\mathrm{f}}$ probes a region outside that most affected by adiabatic contraction.

Warps and non-circular motions are also a potential concern for the present analysis, since in a $\Lambda \mathrm{CDM}$ universe dark matter halos are expected to be triaxial, which would induce noncircular motions in the gas (Hayashi \& Navarro 2006), and gas moving through filaments (Dekel \& Birnboim 2006) will interact with galaxies, triggering structures on discs like those warps. Nearly all the rotation curves collected in the present paper were derived using the tilted-ring fitting of the velocity field, which can account for warps but not for non-circular motions. The exception is DDO 47, which was studied in detail by Gentile et al. (2005), using the harmonic decomposition of the velocity field (Wong et al. 2004). So we exclude that warps might have an effect on the paper conclusions, while we cannot exlude the possibility of non-circular motions; however, one can expect them to increase the observational scatter, but not to have a systematic effect.

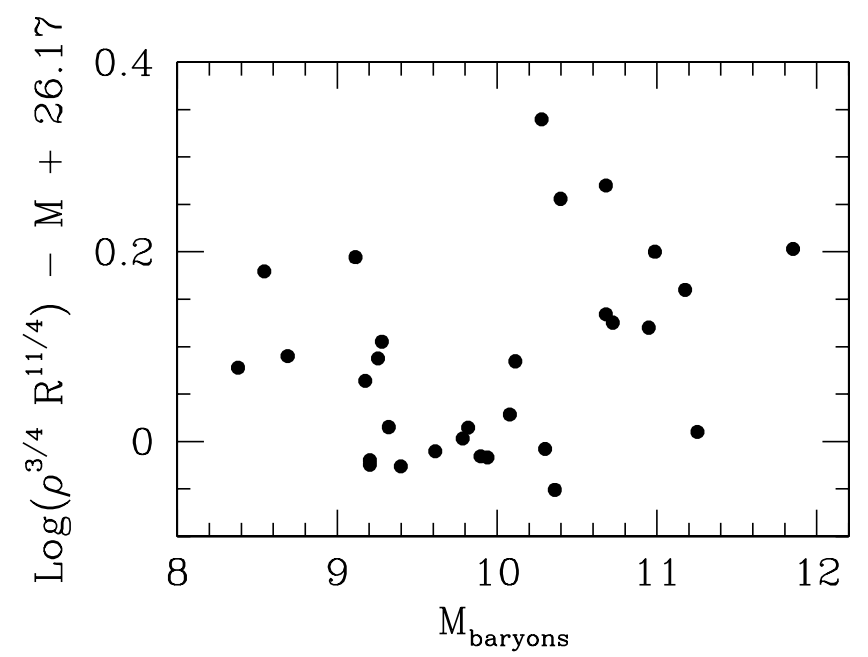

Fig. 6. Distance from Eq. (6) vs. baryonic mass of the galaxies with mass decompositions: there is no clear correlation between the two quantities. Units are the same as in Fig. 3.

The outer density discrepancy arises in a clear way by setting the NFW halo mass within the last point equal to the observed one at $R_{\mathrm{f}}$; what happens if this is valid at a different radius? If the radius is smaller, the density inversion happens at even smaller radii, and the discrepancy at $R_{\mathrm{f}}$ is worse. If the radius is larger, there might be no density inversion, but the cusp/core discrepancy (see however Sect. 1) between $\Lambda$ CDM predictions and observations would be increased and would be present at any radius where baryons are present. The latter case is shown in Fig. 2, where at the last point we imposed $\rho_{\mathrm{h}}\left(R_{\mathrm{f}}\right)=\rho_{\mathrm{NFW}}\left(R_{\mathrm{f}}\right)$ instead of $M_{\mathrm{h}}\left(R_{\mathrm{f}}\right)=M_{\mathrm{NFW}}\left(R_{\mathrm{f}}\right)$ : the inner discrepancy becomes worse. Even though the method of analysis is different, this effect might be related to the results of Seigar et al. (2006), whose NFW models (including possible adiabatic contraction) match the observed rotation curves of two galaxies in their outer parts $(\sim 10 \mathrm{kpc})$, but overestimated the inner rotation curves. One of the two cases shown by Seigar et al. (2006) is a barred galaxy, and its fitted concentration parameter is small compared to the averaged predicted value; the authors discuss possible evidence for the absence of adiabatic contraction or alternatively of some dynamical effects that compensate for adiabatic contraction.

By assuming the Burkert halo instead of the NFW halo leads to the results shown in Fig. 5. Here we consider the one-parameter family of halos defined by the Burkert halo and the empirical relation between the central density $\rho_{0}$ and the core radius $r_{0}$ found by Salucci \& Burkert (2000): $\rho_{0}=3 \times$ $10^{-24}\left(r_{0} / \mathrm{kpc}^{-2 / 3} \mathrm{~g} \mathrm{~cm}^{-3}\right.$. The virial masses were computed by integrating the density profile until the mean density was $\Delta$ times $\rho_{\mathrm{c}}$, where $\Delta$ was derived following Bryan \& Norman (1998). In this case (see Fig. 5), we find that halos with different masses do not overlap (because for the chosen axes there is no such approximation as Eq. (8)), and that the agreement with the observations is much better than in the case of the NFW halos. This means that the Burkert halo, known to fit the inner parts of rotation curves better, is also a better representation of the observations at the last radii probed by rotation curves ( $\sim 5-35 \%$ of the virial radius).

Let us point out that while the present work shows that a theory vs. observations discrepancy extends well beyond the very inner regions of spirals, it leaves open the possibility that, for radii $r \gtrsim 0.2-0.3 r_{\text {vir }}$, the density of the DM around galaxies converges to a NFW profile. This was hinted at by 
Prada et al. (2003), who have investigated the very outer dark matter profile by means of the kinematics of satellites around isolated galaxies. However, they probe an outer region with respect to those considered here and in a very low resolution mode: their projected radii range from 20 to $350 \mathrm{kpc}$, in $100 \mathrm{kpc}$ bins. Brainerd (2004a,b), using weak galaxy lensing in addition to the dynamics of satellite galaxies, reaches similar conclusions for scales $\gtrsim 50 h^{-1} \mathrm{kpc}$. The results shown in the present paper hold for galaxies, but the situation on the scales of galaxy clusters might be different: indeed, Vikhlinin et al. (2006) and Zappacosta et al. (2006) show agreement between X-ray data and $\Lambda$ CDM mass profiles.

\section{Conclusions}

An accurate mass modelling of the external regions in the case of a couple of test-case spirals and a careful determination of the densities and enclosed masses of the dark matter haloes at the farthest radii at which 37 high quality rotation curves have been measured, has brought about the discovery of a new problem/discrepancy for the $\Lambda \mathrm{CDM} / \mathrm{NFW}$ haloes. In fact, in addition to the well-known evidence for which in the inner regions of galaxies $\left(R<2 R_{\mathrm{D}}\right)$ the DM haloes show a flattish density profile, with amplitudes up to one order of magnitude lower than the $\Lambda \mathrm{CDM}$ predictions, at outer radii $\left(R>4 R_{\mathrm{D}}\right)$ the measured $\mathrm{DM}$ halo densities are found to be higher than the corresponding $\Lambda \mathrm{CDM}$ ones. This implies either that the shallow-steep disagreement extends all over 1-2 times the galaxy optical radius (which would be the case if $\rho_{\mathrm{NFW}}\left(R_{\mathrm{f}}\right)=\rho\left(R_{\mathrm{f}}\right)$ ) or that there is a complex data vs. theory disagreement.

While the statistical significance and the level of the discrepancy must be investigated with more and outer data, there is already evidence of this discrepancy in most galaxies with high quality data. The DM halo density, known to have a core in the internal regions, does not seem to converge to the NFW profile at 4-6 $R_{\mathrm{D}}$. This implies an issue for $\Lambda \mathrm{CDM}$ that should be investigated in the future, when, due to improved observational techniques, the kinematic information will be extended to the $\sim 100 \mathrm{kpc}$ scale (Gentile et al., in prep.).

This new discrepancy provides additional information on the nature of the cusp/core issue: self-interacting or annihilating dark matter proposed as the cause for the inner discrepancy may be in difficulty in that it will cause a rapid convergence to the NFW profile in the luminous parts of galaxies and beyond once a critical density value is reached. The discrepancy points to a scenario of modified CDM profiles, to a global mass or angular momentum rearrangement (e.g., Dutton et al. 2006; Tonini et al. 2006) that would remove dark matter from the innermost parts to the radii probed by the outermost regions of rotation curves.

\section{Appendix A: Persic \& Salucci (1990) method to derive the disc mass}

The total velocity profile is given by assuming the total centrifugal equilibrium between the two components, the baryons (only the disc is taken into account, as the bulge just affects the centre of the rotation curve (RC), and the stellar halo mass and the $\mathrm{HI}$ disc are negligible for our purposes) and the dark matter:

$V^{2}(R)=V_{\mathrm{D}}^{2}(R)+V_{\mathrm{H}}^{2}(R)$.

The halo and disc mass inside a given radius $R$ is $M_{\mathrm{H}}(R)=$ $G^{-1} V_{\mathrm{H}}^{2}(R) R$ and the disc contribution to the circular velocity is: $V_{\mathrm{D}}^{2}(R)=G M_{\mathrm{D}} f / R$, respectively, where $f=1 / 2\left(R / R_{\mathrm{D}}\right)^{2}\left(I_{0} K_{0}-\right.$ $\left.I_{1} K_{1}\right)\left.\right|_{R / 2 R_{\mathrm{D}}}$ is defined in terms of the modified Bessel functions
$I_{n}$ and $K_{n}$. Let us now consider the moments of Eq. (A.1), and let us define the logarithmic slopes of the total, disc, and halo velocity, respectively: $\left.\nabla \equiv \frac{\mathrm{d} \log V(R)}{\operatorname{dlog} R}\right|_{R_{\mathrm{opt}}},\left.\nabla \equiv \frac{\mathrm{d} \log V_{\mathrm{D}}(R)}{\operatorname{dlog} R}\right|_{R_{\mathrm{opt}}}=-0.27$, $\left.\nabla_{\mathrm{H}} \equiv \frac{\mathrm{d} \log V_{\mathrm{H}}(R)}{\operatorname{dlog} R}\right|_{R_{\mathrm{opt}}}$, where $R_{\mathrm{opt}}=3.2 R_{\mathrm{D}}$. While $\nabla$ is observed, $\nabla_{\mathrm{H}}$ is unknown, and it is related to the dark matter distribution by: $\mathrm{d}\left(\log M_{\mathrm{H}}(R)\right) / \mathrm{d}(\log R)_{R_{\mathrm{opt}}}=2 \nabla_{\mathrm{H}}+1$. Since $M_{\mathrm{D}} \approx$ $1.1 V_{\mathrm{D}}^{2}(R) R / G$, we can now write the disc-to-total mass ratio at the optical radius $R_{\mathrm{opt}}$ :

$$
\frac{M_{\mathrm{D}}\left(R_{\mathrm{opt}}\right)}{M_{\mathrm{total}}\left(R_{\mathrm{opt}}\right)}=\frac{\nabla_{\mathrm{H}}-\nabla}{\nabla_{\mathrm{H}}+0.11 \nabla+0.30} .
$$

It is reasonable to assume that at the optical radius the halo $\mathrm{RC}$ is not decreasing, and the DM density is not increasing, therefore $0 \leq \nabla_{\mathrm{H}} \leq 1$; in the maximum disc hypothesis, we can find the value of $\nabla_{\mathrm{H}}$ in the interval $[0,1]$ that maximises the ratio of Eq. (A.2): $\frac{\mathrm{d}\left[M_{\mathrm{D}}\left(R_{\mathrm{opt}}\right) / M_{\mathrm{total}}\left(R_{\mathrm{opt}}\right)\right]}{\mathrm{d} \nabla_{\mathrm{H}}}=0$ and in this case it is easy to see that $\nabla_{\mathrm{H}}=1$. From Eq. (A.2) we then obtain:

$M_{\mathrm{D}} \simeq 1.32 V_{\mathrm{opt}}^{2} R_{\mathrm{opt}}(0.77-0.83 \nabla)$,

which allows us to obtain the halo velocity profile at any given point in its luminous and dark contributions, by means of $M_{\mathrm{D}}, f$ and Eq. (A.1).

Notice that this minimum halo assumption used to derive the disc mass might not be completely correct and it might enhance the evidence for an inner core, but it is perfectly legitimate in the present procedure in that in any case, it just (slightly) underestimates the DM density at large radii, bringing even more support to our claim. In any case the disc mass, obtained by means of Eq. (A.3), plays a very minor role in the results of this work, in fact at large distances the mass and density of the dark matter at any outermost radius (denoted as $R_{\mathrm{f}}$ ) do not depend significantly on this quantity. We have, supposing to deal with a system in virial equilibrium:

$\rho\left(R_{\mathrm{f}}\right)=\frac{V^{2}\left(R_{\mathrm{f}}\right)}{4 \pi G R_{\mathrm{f}}^{2}}\left(1+\left.2 \frac{\mathrm{d} \log V(R)}{\operatorname{dlog} R}\right|_{R_{\mathrm{f}}}\right)+\frac{M_{\mathrm{D}}}{R_{\mathrm{D}}^{3}} f\left(R_{\mathrm{f}} / R_{\mathrm{D}}\right)$

(see Fall \& Efstathiou 1980). We realise that, at most, the second term is a correction of $30 \%$. So errors (even quite large) in the estimate of $M_{\mathrm{D}}$ propagate little in the estimate of $\rho\left(R_{\mathrm{f}}\right)$.

\section{References}

Barnes, E. I., Sellwood, J. A., \& Kosowsky, A. 2004, AJ, 128, 2724 Bell, E. F., McIntosh, D. H., Katz, N., \& Weinberg, M. D. 2003, ApJS, 149, 289 Blais-Ouellette, S., Amram, P., \& Carignan, C. 2001, AJ, 1952, 1964

Blais-Ouellette, S., Amram, P., Carignan, C., \& Swaters, R. 2004, A\&A, 420, 147

Brainerd, T. G. 2004a, preprint [arXiv: astro-ph/0409381]

Brainerd, T. G. 2004b, The New Cosmology: Conference on Strings and Cosmology, AIP Conf. Proc., 743, 129

Blumenthal, G. R., Faber, S. M., Flores, R., \& Primack, J. R. 1986, ApJ, 301, 27 Borriello, A., \& Salucci, P. 2001, MNRAS, 323, 285

Bosma, A., Byun, Y., Freeman, K. C., \& Athanassoula, E. 1992, ApJ, 400, L21 Bottema, R. 1999, A\&A, 348, 77

Bryan, G.-L., \& Norman, M.-L. 1998, ApJ, 495, 80

Burkert, A. 1995, ApJ, 447, L25

Carignan, C., Charbonneau, P., Boulanger, F., \& Viallefond, F. 1990, A\&A, 234, 43

de Blok, W. J. G. 2005, ApJ, 634, 227

de Blok, W. J. G., \& Bosma, A. 2002, A\&A, 385, 816

de Blok, W. J. G., Bosma, A., \& McGaugh, S. 2003, MNRAS, 340, 657

Dekel, A., \& Birnboim, Y. 2006, MNRAS, 368, 2

Donato, F., Gentile, G., \& Salucci, P. 2004, MNRAS, 353, L17 
Dutton, A. A., Courteau, S., de Jong, R., \& Carignan, C. 2005, 619, 218

Dutton, A. A., van den Bosch, F. C., Dekel, A., \& Courteau, S. 2006, ApJ, 654, 27

Gentile, G., Salucci, P., Klein, U., Vergani, D., \& Kalberla, P. 2004, MNRAS, 351,903

Gentile, G., Burkert, A., Salucci, P., Klein, U., \& Walter, F. 2005, ApJ, 634, L145 Gentile, G., Salucci, P., Klein, U., \& Granato, G. L. 2007, MNRAS, 375, 199 Giraud, E. 1998, AJ, 116, 2177

Gnedin, O. Y., Kravtsov, A. V., Klypin, A. A., \& Nagai, D. 2004, ApJ, 616, 16

Gnedin, O. Y., Weinberg, D. H., Pizagno, J., Prada, F., \& Rix, H.-W. 2006, preprint [arXiv:astro-ph/0607394]

Gustafsson, M., Fairbairn, M., \& Sommer-Larsen, J. 2006, Phys. Rev. D, 74, 123522

Hayashi, E., \& Navarro, J. F. 2006, MNRAS, 373, 1117

Kazantzidis, S., Kravtsov, A. V., Zentner, A. R., et al. 2004, ApJ, 611, L73

McGaugh, S. S., de Blok, W. J. G., Schombert, J. M., et al. 2007, ApJ, 659, 149

Navarro, J. F., Frenk, C. S., \& White, S. D. M. 1996, ApJ, 462, 563

Noordermeer, E., van der Hulst, T., \& Swaters, R. 2004, Proceedings of Baryons in Dark Matter Halos. Novigrad, Croatia, 5-9 Oct. 2004, ed. R. Dettmar, U. Klein, P. Salucci, published by SISSA, Proceedings of Science, [http://pos.sissa.it], 68

Persic, M., \& Salucci, P. 1990, MNRAS, 247, 349

Prada, F., Vitvitska, M., Klypin, A., et al. 2003, ApJ, 598, 260

Salucci, P., \& Burkert, A. 2000, ApJ, 537, L9

Salucci, P., Walter, F., \& Borriello, A. 2003, A\&A, 409, 53
Seigar, M. S., Bullock, J. S., Barth, A. J., \& Ho, L. C. 2006, ApJ, 645, 1012

Sellwood, J. A., \& McGaugh, S. S. 2005, ApJ, 634, 70

Simon, J. D., Bolatto, A. D., Leroy, A., Blitz, L., \& Gates, E. L. 2005, ApJ, 621, 757

Sofue, Y., Tutui, Y., Honma, M., et al. 1999, ApJ, 523, 136

Spergel, D. N., Bean, R., Doré, O., et al. 2006, preprint [astro-ph/0603449] Swaters, R. A., Madore, B. F., van den Bosch, F. C., \& Balcells, M. 2003, ApJ, 583,732

Tonini, C., Lapi, A., \& Salucci, P. 2006, ApJ, 649, 591

Valenzuela, O., Rhee, G., Klypin, A., et al 2007, ApJ, 657, 773

van Albada, T. S., Bahcall, J. N., Begeman, K., \& Sancisi, R. 1985, ApJ, 295, 305

van den Bosch, F. C., Robertson, B. E., Dalcanton, J., \& de Blok, W. J. G. 2000, AJ, 119, 1579

van Zee, L., \& Bryant, J. 1999, AJ, 118, 2172

Verheijen, M. A. W., \& Sancisi, R. 2001, A\&A, 370, 765

Vikhlinin, A., Kravtsov, A., Forman, W., et al. 2006, ApJ, 640, 691

Walsh, W., Staveley-Smith, L., \& Oosterloo, T. 1997, AJ, 113, 1591

Wechsler, R. H., Bullock, J. S., Primack, J. R., Kravtsov, A. V., \& Dekel, A. 2002, ApJ, 568, 52

Weiner, Benjamin, J., Williams, T. B., van Gorkom, J. H., \& Sellwood, J. A. 2001, ApJ, 546, 916

Weldrake, D. T. F., de Blok, W. J. G., \& Walter, F. 2003, MNRAS, 340, 12

Wong, T., Blitz, L., \& Bosma, A. 2004, ApJ, 605, 183

Zappacosta, L., Buote, D. A., Gastaldello, F., et al. 2006, ApJ, 650, 777 